\title{
A PROCEDURE FOR THE REGISTRATION AND SEGMENTATION OF HETEROGENEOUS LIDAR DATA
}

\author{
Al-Durgham M. ${ }^{\mathrm{a}}$, Habib A. ${ }^{\mathrm{b}}$ \\ ${ }^{a}$ Dept. of Civil Engineering, University of Toronto, 35 St. George Street, Toronto, ON, M5S 1A4, Canada \\ ${ }^{\mathrm{b}}$ Dept. of Geomatics Engineering, University of Calgary, 2500 University Drive, Calgary, AB, T2N 1N4, Canada \\ a mohannad.al.durgham@utoronto.ca, bahabib@ucalgary.ca
}

Commission I, WG I/3

KEY WORDS: LiDAR, Registration, Segmentation, Fusion, Automation

\begin{abstract}
:
Laser scanning, whether airborne or terrestrial is being used nowadays for wide spectrum of applications. In addition, many advances have been introduced to the laser scanning technology in the last decade; thus resulting into increased performance in terms of the point density, scanner range, and expected point accuracy. On the other hand, users are encountering scenarios where the integration of various laser datasets becomes essential in order to avoid data gaps (e.g., missing building roofs in the terrestrial scans, or missing structure facades in the airborne case). This problem is usually solved seamlessly through a classical transformation when the average point accuracy is relatively homogeneous over a given dataset. However, this is not usually the case; in this work, we propose a workflow for the optimal registration of multisource point clouds using weighted conformal transformation. First, the individual scans are filtered and the local point attributes are populated through a data characterization step. Then, an ICPP-based weighted registration algorithm is performed over the entire datasets until convergence. Finally, our heterogeneous segmentation procedure is performed in a simultaneous fashion to ensure exploiting the full potential of a dataset. The performance of this algorithm in terms of correctness, automation level, and other factors is evaluated using real datasets with significant variations in point densities and accuracy.
\end{abstract}

\section{INTRODUCTION}

LiDAR data registration and segmentation are considered amongst the first steps in data post-processing. The accuracy of these phases highly impacts the expected quality of the final product. Improper registration and/or segmentation will result into artifacts when attempting to reconstruct building models for example. Although many algorithms have been proposed for the registration and segmentation of LiDAR datasets, the compatibility between these datasets is often overlooked. In this work, we propose a procedure for augmenting heterogeneous LiDAR datasets in an optimal fashion while taking into account the data quality variations.

Segmentation of LiDAR data is useful for multiple applications: (1) in terrain extraction (Tovari \& Pfeifer, 2005), where a segmentation algorithm is used to perform a ground vs. nonground separation, (2) in building detection (Kim et al., 2007), where mostly planar patches that are found above ground level are extracted, and finally (3) in data filtering (Sithole \& Vosselman, 2005) where non-planar points are identified as outliers.

To the best of the authors' knowledge, there is no literature that addresses the segmentation of heterogeneous Laser data. However, any of the currently existing segmentation may be extended to accommodate heterogeneous datasets. Please refer to Vosselman \& Maas, 2010 and Shan \& Toth, 2008 for variants of segmentation algorithms. In this paper, we present our strategy of expanding our region growing based segmentation algorithm to take into account scans with varying characteristics.

First, the individual scans are filtered and the individual point attributes are updated using a data characterization procedure. Afterwards, the weighted iterative closest projected point (ICPP) registration algorithm is performed over the entire datasets until convergence. The proper weightings in this registration step are derived from the surface roughness evaluated during the aforementioned characterization procedure.

Once the datasets are adequately registered, we employ the attributes collected in the first step such as the local point densities, approximate surface roughness, and surface normal directions. In the Heterogeneous segmentation step, a weighted least squares and a region-growing-based algorithm is adopted. In similar fashion to the registration, weighting of individual points is established using the surface roughness computed in the data characterization step.

Using the proposed combined segmentation is beneficial for multiple reasons: (1) missing or incomplete features in one or more scans are likely to be complete in the combined dataset, (2) the overall increased point density is very beneficial for detecting finer planar patches, and the weighted least squares will ensure that the integrity of the combined dataset is maintained, and (3) The results of the combined segmentation is useful to verify the correctness of the registration procedure.

In the next section, the heterogeneous registration and segmentation algorithms are presented. Then, we examine our algorithms against a collection of airborne and terrestrial laser scans. Next a discussion of the algorithm's performance is presented. Finally, we conclude this paper with our findings and future research plans.

\section{METHODOLOGY}

Our algorithm consists of three main steps as shown in Figure 1. First a data characterization step is performed to compute individual point attribute. Then the Iterative Closest Projected Point (ICPP) algorithm is used to ensure the alignment between the scans in question. Finally the heterogeneous segmentation procedure is performed over the available scans. In the following subsections, we discuss these steps in more details. 


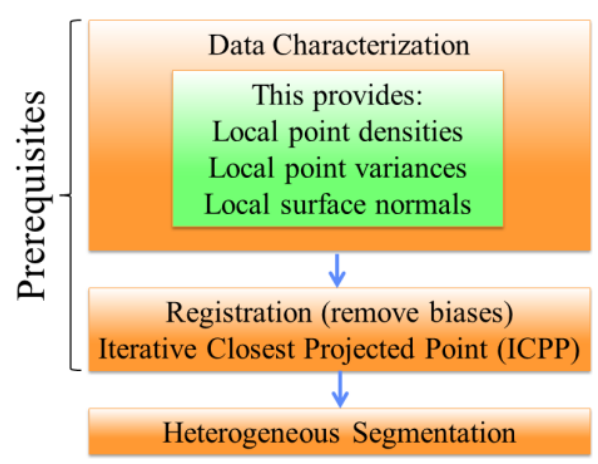

Figure 1: The flow of the segmentation procedure

\subsection{Data characterization step}

This step is performed to compute individual point attributes. Namely, the local point density, local surface roughness, and local surface normal are estimated. Furthermore, planar points are identified and are only used in the following registration step. Point originating from tree crowns and other vegetated areas will negatively impact the quality of the registration procedure since these points might have slightly moved between consecutive scans. This characterization step is identical in concept to a region growing segmentation algorithm performed over a single scan. First, a set of points is selected randomly as seed points and will be used to perform the region growing procedure shown in Figure 2. These seed points are used to perform the region growing procedure.

First, the seed's neighbours are examined to check if they belong to a relatively flat (planar) surface. Then, if the aposteriori variance factor of the plane fitting results is within a predefined range, the neighbours are considered to constitute a planar surface and the region growing procedure begins. While preforming the region growing, one can also estimate the local point density for each point that is visited. Also, the a-posteriori variance factor of the plane fitting and the normal direction of this plane are used as the local surface roughness and approximate normal direction for the points belonging to the plane in question. Note that for every point added to the plane, one must update the plane parameters; this could be achieved through a sequential least squares solution (Sayed, 2003).

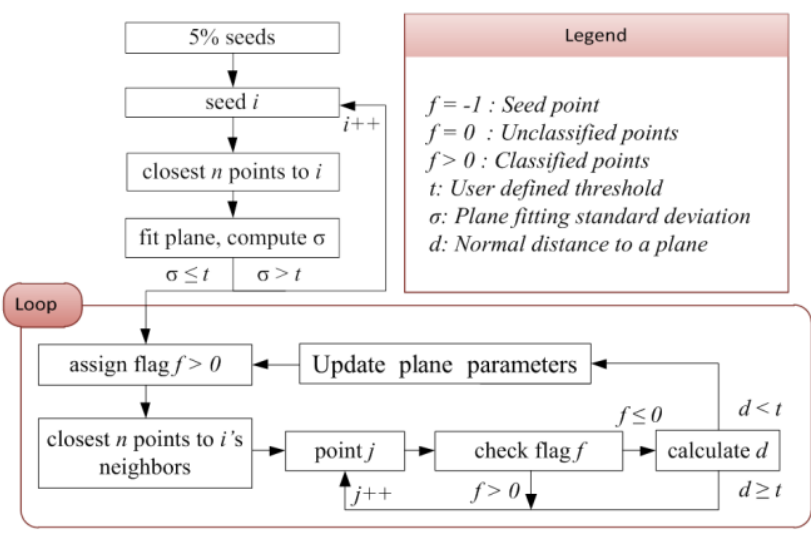

Figure 2: A flowchart summarizing the data characterization procedure.

\subsection{Data registration}

Let us first consider a pairwise registration scenario, where the scans $S_{1}=\left(\boldsymbol{X}_{1}, \boldsymbol{Y}_{1}, \boldsymbol{Z}_{1}\right)$ and $S_{2}=\left(\boldsymbol{X}_{2}, \boldsymbol{Y}_{2}, \boldsymbol{Z}_{2}\right)$ are transformed to a predefined reference frame $(r)$. In this case, the transformation mathematical model takes the following form:

$$
0=\boldsymbol{T}_{2}^{r}+\boldsymbol{R}_{2}^{r} \boldsymbol{p}^{\prime}-\left(\boldsymbol{T}_{1}^{r}+\boldsymbol{R}_{1}^{r} \boldsymbol{p}\right)
$$

$\boldsymbol{T}_{1}^{r}$, is the translation vector between $S_{1}$ and a predefined reference frame

$\boldsymbol{R}_{1}^{r}$, is the rotation matrix between $S_{1}$ and a predefined reference frame

$\boldsymbol{T}_{2}^{r}$, is the translation vector between $S_{2}$ and a predefined reference frame

$\boldsymbol{R}_{2}^{r}$, is the rotation matrix between $S_{2}$ and a predefined reference frame

$\boldsymbol{p}$, is a point in $S_{1}$

$\boldsymbol{p}^{\prime}$, is the projection of $\boldsymbol{p}$ into $S_{2}$ as will be explained later on.

One could manipulate Eq. 1 to calculate the transformation of $\boldsymbol{p}$ into $S_{2}$. Then, $\boldsymbol{p}^{\prime}$ could be derived by computing the projection of the transformed point into the closest triangle formed by three points in $S_{2}\left(\boldsymbol{p}_{1}, \boldsymbol{p}_{2}, \boldsymbol{p}_{3}\right)$ as shown in Figure 3 . Then, the normal distance $N$ is examined and if it falls within a predefined threshold then the pair will be used in the registration. For more details about this algorithm, please refer to Al-Durgham et al., 2011. Note that the ICPP explained in Al-Durgham et al., 2011 does not account for weighting of individual points, thus we introduce the weightings proportional to the local surface roughness of the point in question.

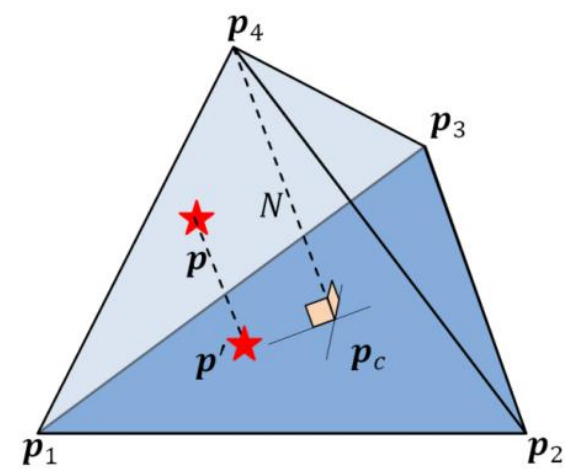

Figure 3: The ICPP point to triangle matching

\subsection{Heterogeneous segmentation}

To better demonstrate this algorithm, consider three overlapping laser scans A, B, and C. Seed points are first established over all the scans. Then, starting with scan A, one finds a group of points that are planar in nature. When a plane is found in scan $\mathrm{A}$, the region growing is executed until no point that belongs to the plane in question is found. However, note that due to the shadowing cause by other objects, the region growing might have prematurely terminated. Next, the identified points in scan $\mathrm{A}$ are used as seeds to query scan B and scan $\mathrm{C}$. The region growing is then performed across those two scans in similar fashion until the region growing is complete as shown in Figure 4.

Note that, the new points that were found in scans B and C are used in return to query scan A for new points. This recursive process of investigating scans over and over as new points are discovered continues until no additional points are found to belong to the current plane. The recursive least squares formulas for updating the plane parameters are similar to those shown in (Sayed, 2003) while introducing individual weightings of the added points. 


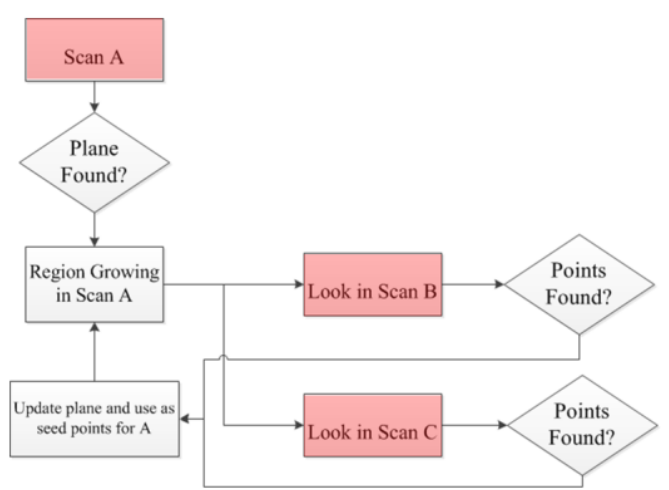

Figure 4: A flowchart demonstrating the heterogeneous segmentation

\section{DATA USED}

Our heterogeneous segmentation algorithm was tested using a dataset with significantly varying characteristics. A set of fourteen tripod-mounted laser scans were collected over the Yamnuska at the University of Calgary. The terrestrial laser scans available for processing have a point density of 200 $\mathrm{pts} / \mathrm{m}^{2}$. In addition, a set of five laser scans with varying characteristics are available for this experiment. One should mention that the ratio in the point density between the airborne and terrestrial data is significantly high (i.e., 1:80). Table 1 summarizes the average point density and accuracy for this dataset. Note that the reported accuracy in the table is estimated using a characterization process which reflects the surface roughness property and not the actual accuracy of the sensor used.

Table 1: A summary of the scans used and their characteristics

\begin{tabular}{|c|c|c|}
\hline Scan name & $\begin{array}{c}\text { Average point } \\
\text { density }\left(p t s / m^{2}\right)\end{array}$ & $\begin{array}{c}\text { Average point accuracy }(m) \\
\text { (along the plane normal) }\end{array}$ \\
\hline $\begin{array}{c}\text { Stations } \\
1-13\end{array}$ & 200 & $<0.03$ \\
\hline $\begin{array}{c}\text { Airborne } \\
1-5\end{array}$ & 2.5 & $\sim 0.09$ \\
\hline
\end{tabular}

\section{RESULTS AND DISCUSSION}

This section is divided into two subsections. First, we visually evaluate the performance of our registration algorithm over different areas of the datasets. Then, the segmentation performance is evaluated in the second subsection.

\subsection{Registration results}

Figures 5-7 show few snapshots of the dataset. Figure 5 shows a structural column before (a) and after (b) the ICPP algorithm has been performed. Note how the registration quality among the terrestrial scans is not affected by the degraded quality of the airborne datasets. The points appearing inside the cylinder are caused by features located ontop of the column. Figure 6 shows a cross section of a building rooftop together with a ground segment. This figure demonstrates the agreement between terrestrial and airborne data.

Figure 7 shows another example of the registration quality between the airborne and terrestrial datasets as appearing in the ground segment. The orange-coloured points appearing in the right side of the graph are the result of a known artefact in the laser scanning mechanism known as the mixed pixel problem (Lichti et al., 2005).

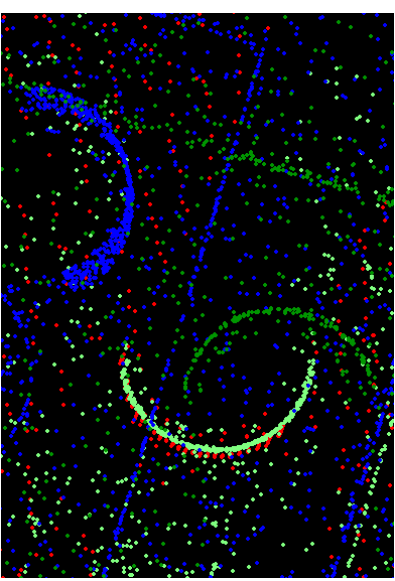

(a)

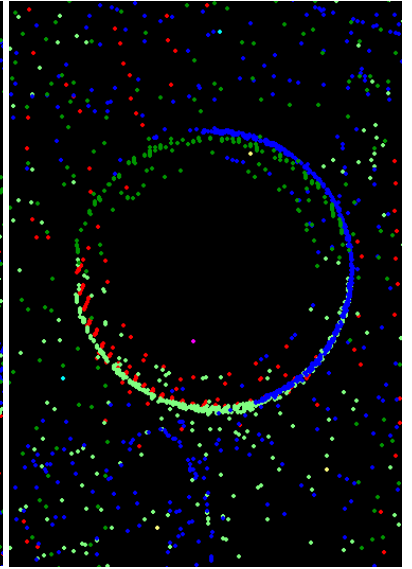

(b)
Figure 5: A cross section of a cylinder-shaped structural column before (a) and after (b) running the ICPP.

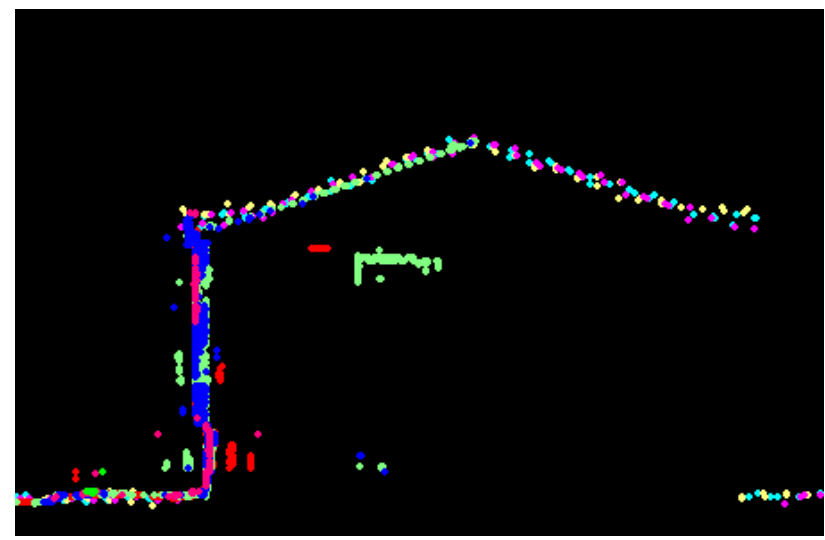

Figure 6: A cross section of a gable roof and a ground segment demonstrating the alignment between the airborne and terrestrial data

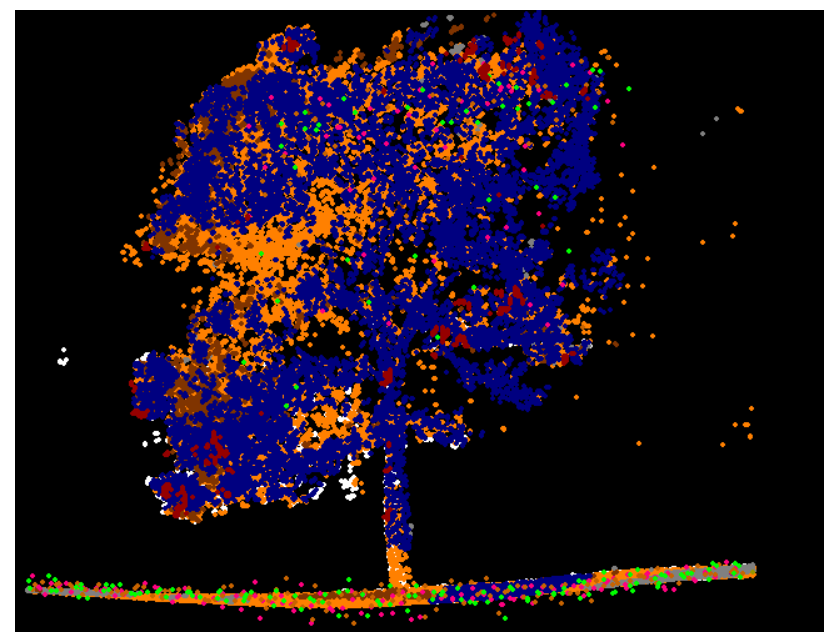

Figure 7: A tree crown and a ground segment with points originating from multiple airborne and terrestrial scans

\subsection{Heterogeneous segmentation results}

Figure 8(a) shows a top view of the heterogeneous segmentation results. One can clearly see the significant variations in the point density between the Yamnuska Hall rooftop and the 
terrain. Figure 8(b) show another view of the same structure where the variations in the point density are more visible.

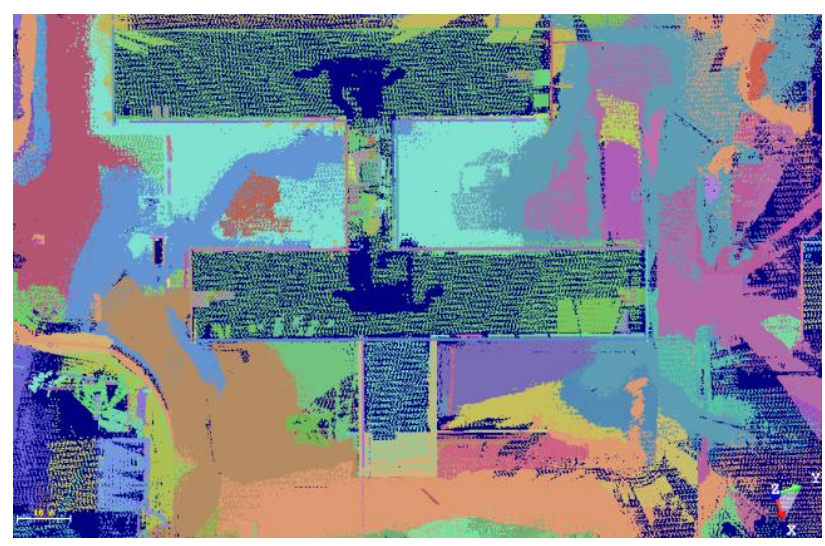

(a)

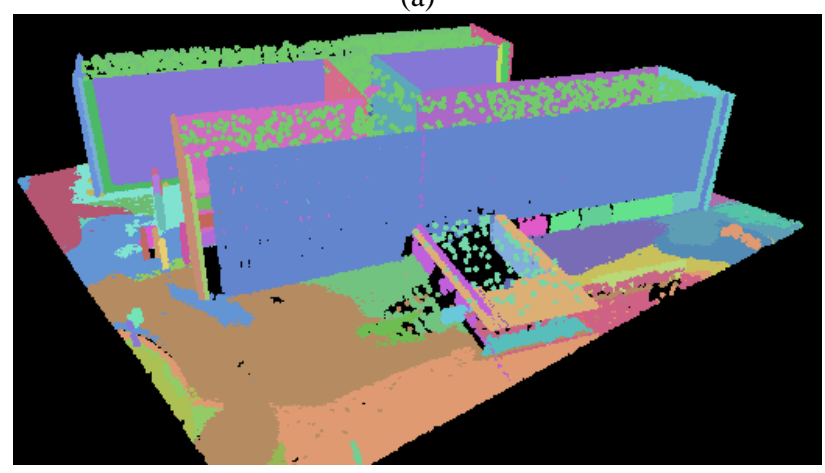

(b)

Figure 8: A top view of the Yamnuska Hall segmented point cloud (a) and an isometric view of the same structure.

Finally, Figure 9 shows a cross section of the Yamnuska Hall entrance. This figure demonstrates the advantage of assigning point accuracies based on the surface roughness as opposed to assigning a single accuracy value over the entire dataset.

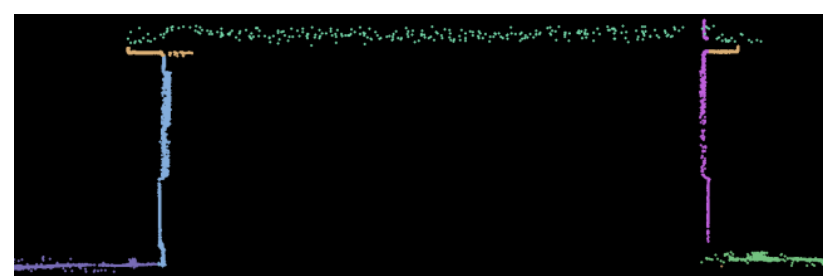

Figure 9: A cross section of a structure showing the segmented terrestrial and airborne scans

\section{CONCLUDING REMARKS AND FUTURE WORK}

In this paper, a framework for the simultaneous post processing of heterogeneous LiDAR data has been proposed. In particular, the registration and segmentation of airborne and terrestrial LiDAR data in an optimal fashion has been addressed. The local surface roughness of a point has been used as an alternative for the point accuracy. The registration results demonstrated accurate alignment between heterogeneous laser scans. The heterogeneous segmentation proposed shows that one can overcome the segmentation artefacts caused by variation in the local point density.
Future work will be focused on generating building models from the heterogeneous datasets. Digital building models are expected to be more complete in a heterogeneous dataset due to the useful view angle variations. In addition, the integration of the LiDAR and other point-based datasets such as point clouds resulting from imagery using the semi-global matching algorithm is of interest.

\section{ACKNOWLEDGMENT}

The authors would like to thank Jean-Sebastien GauthierMathieu, Brett Vaile, Kale Pominville, and Natasha Wong Ken from the University of Calgary for performing the data acquisition. In addition, we are grateful to Prof. Brenda McCabe, Department of Civil Engineering, University of Toronto for supporting this work.

Also, sincere thanks go to the GEOIDE NCE network (project: PIV_SII72), NSERC Discovery and Strategic Project Grants, Tecterra for their financial support of this research. Last but not least, the authors are in debt to the Canadian Foundation of Innovation for funding the purchase of the laser scanner used in this research.

\section{REFERENCES}

Al-Durgham, M., Detchev, I. \& Habib, A., 2011. Analysis of Two Triangle-Based Multi-Surface Registration Algorithms of Irregular Point Cloud. In ISPRS Workshop Laser Scanning. Calgary, 2011.

Kim, C., Habib, A. \& Mrstik, P., 2007. New approach for planar patch segmentation using airborne laser data. In ASPRS Annual Conference., 2007.

Lichti, D., Gordon, S. \& Tipdecho, T., 2005. Error Models and Propagation in Directly Georeferenced Terrestrial Laser Scanner Networks. Journal of Surveying Engineering, 131(4), pp.135-42.

Sayed, A.H., 2003. Fundamentals of Adaptive Filtering. Wiley.

Shan, J. \& Toth, C., 2008. Topographic Laser Ranging and Scanning: Principles and Processing. 1st ed. CRC Press.

Sithole, G. \& Vosselman, G., 2005. Filtering of airborne laser scanner data based on segmented point clouds. International Archives of Photogrammetry,Remote Sensing and Spatial Information Sciences, 36(Part 3/W19), p.66-71.

Tovari, D. \& Pfeifer, N., 2005. Segmentation based robust interpolation - a new approach to laser data filtering. International Archives of Photogrammetry Remote Sensing and Spatial Information Sciences, 36(3/W19), pp.79-84.

Vosselman, G. \& Maas, H.G., eds., 2010. Airborne and Terrestrial Laser Scanning. 1st ed. CRC Press. 